\title{
Simulation algorithms for the random-cluster model
}

\author{
Xiaofeng Qian, ${ }^{1}$ Youjin Deng, ${ }^{2}$ and Henk W. J. Blöte ${ }^{2,1}$ \\ ${ }^{1}$ Lorentz Institute, Leiden University, P.O. Box 9506, 2300 RA Leiden, The Netherlands \\ ${ }^{2}$ Faculty of Applied Sciences, Delft University of Technology, P.O. Box 5046, 2600 GA Delft, The Netherlands
}

(Received 16 August 2004; published 28 January 2005)

\begin{abstract}
We compare the performance of Monte Carlo algorithms for the simulation of the random-cluster representation of the $q$-state Potts model for continuous values of $q$. In particular we consider a local bond update method, a statistical reweighting method of percolation configurations, and a cluster algorithm, all of which generate Boltzmann statistics. The dynamic exponent $z$ of the cluster algorithm appears to be quite small, and to assume the values of the Swendsen-Wang algorithm for $q=2$ and 3. The cluster algorithm appears to be much more efficient than our versions of the other two methods for the simulation of the random-cluster model. The higher efficiency of the cluster method with respect to the local method is primarily due to the fact that the computer time usage of the local method increases more rapidly with system size; the difference between the dynamic exponents is less important.
\end{abstract}

DOI: 10.1103/PhysRevE.71.016709 PACS number(s): 05.10.-a, 05.50.+q, 64.60.-i, 75.10.Hk

\section{INTRODUCTION}

The Potts model [1], which can be seen as a generalization of the Ising model, has been the subject of considerable research in recent decades [2]. Many of these investigations make use of the Kasteleyn-Fortuin mapping on the randomcluster model [3]. Remarkably, the symmetry parameter $q$ of the $q$-state Potts model appears as a continuous parameter in the random-cluster model. Thus the random-cluster model is a generalization of the Potts model to noninteger values of $q$ (and on this basis one might even choose to refer to such a model with noninteger $q$ as a Potts model). For the integer $q=1$, the random-cluster model reduces to the bondpercolation model.

Another mapping, formulated by Baxter, Kelland, and Wu [4], leads from the random-cluster model on a planar lattice to the six-vertex model, which is a limiting case of Baxter's eight-vertex model [5]. This second mapping lends further physical meaning to the random-cluster model.

While many questions concerning the random-cluster model could be answered exactly [6,7], in many cases, especially in more than two dimensions, numerical approximations are needed. For integer $q>1$ one can obviously apply a Metropolis-type algorithm to the Potts representation of the model. However, such simulations suffer from the criticalslowing-down phenomenon, which inhibits the investigation of relatively large system sizes. This problem was partly solved by Swendsen and Wang [8]. Their algorithm is nonlocal in the sense that arbitrarily large groups of Potts variables are flipped at the same time. As a result, critical slowing down, as expressed by the dynamic exponent $z$, is reduced, though not eliminated. The dynamic exponent is still dependent on the number of states $q$ and the dimensionality $d$, as reviewed in Ref. [9].

Simulation methods have been developed as well for noninteger $q$. While the random-cluster model is rich and interesting in its own right, the work on such algorithms for general $q$ may further be justified by fundamental questions such as whether the renormalization scenario [10] for the twodimensional Potts model does also apply in more than two dimensions. Although this question can also be studied by means of analytical approximations [11,12], their accuracy is difficult to estimate and numerical tests are thus desirable. Furthermore, some critical exponents, for instance, the socalled backbone exponent of the Potts model is not exactly known, even in two dimensions. It may be determined numerically as a function of $q$ by means of Monte Carlo methods, and then it is natural to include noninteger values of $q$ [13] for a more complete coverage.

A local Monte Carlo algorithm for the noninteger $q$ random-cluster model in two dimensions was formulated by Sweeny [14]. It updates individual bond variables. Although it has been reported that critical slowing down is absent [15], the Li-Sokal method [16] is also applicable here and it follows that $z \geqslant \alpha / \nu$ where $\alpha$ and $\nu$ are the specific-heat and correlation-length exponents, respectively. Therefore systems with a positive specific-heat exponent $\alpha$ must display critical slowing down, as has been confirmed later [17]. In this algorithm, the transition probabilities depend on nonlocal information: whether neighbor sites are connected by a percolating path of bond variables. Thus the execution of a bond update may require the exploration of a large percolation cluster. Since the pertinent cluster size is divergent at criticality, the number of operations needed for an update of the system increases faster than the number of sites $N$ in the system. How much faster it increases still depends on the sophistication of the algorithm; the Sweeny [14] algorithm is relatively efficient because it avoids the formation of a whole cluster by following only its perimeter instead.

In a different approach, Hu [18] applied a statistical reweighting procedure to bond percolation configurations in order to sample the $q \neq 1$ random-cluster model. While this model has no critical slowing down in the sense that it generates uncorrelated configurations, the number of samples needed before a significant weight occurs increases rapidly with the system size [19]. In practice, this effect is similar to critical slowing down in the sense that many simulation steps have to be performed before a meaningful new sample is obtained. 
Given the recent simulations $[13,15]$ that have been performed using the local bond update method, it would be interesting to compare with the performance of a cluster algorithm for continuous $q$ random-cluster models. Indeed the Swendsen-Wang algorithm can be adapted to include noninteger values of $q$; such an algorithm was described by Chayes and Machta [20]. The resulting cluster algorithm is simple, and requires only of order $N$ operations for an update of the system. But it is applicable only for $q \geqslant 1$.

In this work we report a comparison between our versions of these three algorithms for noninteger values of $q$. We illustrate their performance by means of simple applications, and we estimate the dynamic exponent of the cluster algorithm for three values of $q$. We feel that our findings may be of some use for those planning numerical investigations of the random-cluster model. In Sec. II we summarize the algorithms, and we report our results in Sec. III.

\section{ALGORITHMS}

For the convenience of the reader, we summarize the three algorithms for the simulation of the random-cluster model. To expose the close relation with the discrete- $q$ Potts model, we start from the Potts partition sum

$$
Z_{\sigma}=\left[\prod_{i=1}^{N} \sum_{\sigma_{i}=1}^{q}\right] \prod_{\langle i j\rangle} \exp \left(K \delta_{\sigma_{i} \sigma_{j}}\right),
$$

where the $\sigma_{i}$ are site variables, and the second product is over all nearest-neighbor pairs $\langle i j\rangle$. The coupling $K$ includes a factor $1 / k_{B} T$ and is restricted to $K \geqslant 0$. The mapping on the random-cluster model [3] eliminates the site variables $\sigma_{i}$ $=1,2, \ldots, q$ after introducing bond variables $b_{i j}=0$ or 1 between neighboring sites $i$ and $j$. Bonds $b_{i j}=1(0)$ are considered to be present (absent). In terms of the new variables one obtains the random-cluster partition sum

$$
Z_{\sigma}=Z_{b} \equiv\left[\prod_{\langle i j\rangle} \sum_{b_{i j}=0}^{1}\right] q^{n_{c}} u^{n_{b}}=\sum_{\{b\}} \prod_{k=1}^{n_{c}} q u^{n_{b}^{(k)}},
$$

where $u \equiv e^{K}-1, n_{b}$ is the number of present bonds, and $n_{c}$ the number of clusters (or components) formed by these bonds. The sum on $\{b\}$ is shorthand for the sum on all bond variables, and $n_{b}^{(k)}$ is the number of nonzero bonds in the $k$ th cluster.

Equation (2) can serve directly to formulate a Metropolistype importance-sampling algorithm for local updates of the bond variables $b_{i j}$. A bond $\left(b_{i j}=1\right)$ contributes a reduced (i.e., divided by $\left.k_{B} T\right)$ energy $\ln (1 / u)$ if sites $i$ and $j$ are already connected by some other path of such bonds, or $\ln (q / u)$ if they are not connected. Thus the local update of a bond variable requires the performance of a task that is essentially nonlocal: to determine whether $i$ and $j$ belong to the same cluster. After completion of this task, the energy change due to the bond "flip" is known, and thereby the transition probabilities. Given the time-consuming nature of the task mentioned, one naturally avoids it if not necessary [15]. The latter possibility arises if the value of the random number used for the bond update is such that the result $\left(b_{i j}=0\right.$ or 1$)$ does not depend on whether $i$ and $j$ are connected.

In the statistical reweighting method as formulated by $\mathrm{Hu}$ [18] one generates independent configurations of bond variables using the percolation model. This ensemble of configurations can be described by Eq. (2) with $q=1$. The bond probability is $p=u /(u+1)$. Thus the probability distribution of $\{b\}$ is

$$
P_{q=1}(\{b\})=p^{n_{b}}(1-p)^{N_{b}-n_{b}}=u^{n_{b}} / Z_{q=1},
$$

with

$$
Z_{q=1}=\left[\prod_{\langle i j\rangle} \sum_{b_{i j}=0}^{1}\right] u^{n_{b}}=(1+u)^{N_{b}},
$$

where $N_{b}$ is the total number of nearest-neighbor bonds in the system. The expectation value of an observable $A$ depending on $\{b\}$ is, in the random-cluster model,

$$
\langle A\rangle_{R C}=\left[\prod_{\langle i j\rangle} \sum_{b_{i j}=0}^{1}\right] A q^{n^{c}} u^{n_{b}} / Z_{b} .
$$

This can be rewritten as

$$
\langle A\rangle_{R C}=\frac{\left[\prod_{\langle i j\rangle} \sum_{b_{i j}=0}^{1}\right] A q^{n_{c}} u^{n_{b}}}{Z_{q=1}} \times \frac{Z_{q=1}}{Z_{b}}=\left\langle A q^{n_{c}}\right\rangle_{P} /\left\langle q^{n_{c}}\right\rangle_{P},
$$

where the subscript $P$ denotes averaging on percolation configurations generated by Eq. (3). The advantage of this method is that the relevant quantities $A q^{n_{c}}$ and $q^{n_{c}}$ can be sampled on the basis of percolation configurations which are uncorrelated, and simple to generate. The disadvantage is that the reweighting factor $q^{n_{c}}$ can vary, in particular for large system sizes, over such a large range that, among the generated configurations $\{b\}$, those which contribute significantly to the $\langle\cdots\rangle_{P}$ averages become very scarce [19].

The cluster algorithm can conveniently be described in terms of a mapping between the random-cluster model, Eq. (2), and a model with site as well as bond variables. To this purpose one defines auxiliary "color" variables $\widetilde{t}_{k}=0$ or 1 for each cluster $k=1,2, \ldots, n_{c}$ :

$$
Z_{b}=\sum_{\{b\}} \prod_{k=1}^{n_{c}} \sum_{\tilde{t}_{k}=0}^{1} u^{n_{b}^{(k)}\left(1-\tilde{t}_{k}\right)}\left[(q-1) u^{n_{b}^{(k)}}\right]^{\tilde{k}_{k}} .
$$

Clusters of color 0 and 1 have weight 1 and $q-1$, respectively. The sum over the colors can be replaced by a sum over $N$ site-color variables $t_{i}=0$ or 1 if, at the same time, one includes a factor $\delta_{t_{i} t_{j}}^{b_{i j}}$ (with the convention $0^{0}=1$ ) for each bond variable, so that all sites in one cluster have the same color:

$$
Z_{b}=Z_{t b} \equiv \sum_{\{t\}} \sum_{\{b\}} \prod_{\langle i j\rangle}\left(u \delta_{t_{i} t_{j}}\right)^{b_{i j}} \prod_{k=1}^{n_{c}}(q-1)^{t_{s(k)}},
$$

where $s(k)$ is a site in the $k$ th cluster. For a given site configuration $\{t\}$ one distinguishes three types of bonds $(i j)$ :

$$
\text { type } 0: t_{i}=t_{j}=0 \text {; }
$$




$$
\begin{aligned}
& \text { type } 1: t_{i}=t_{j}=1 ; \\
& \text { type } 2: t_{i}+t_{j}=1 .
\end{aligned}
$$

Accordingly, superscripts are appended to the pertinent summation and product signs

$$
\begin{aligned}
Z_{t b}= & \sum_{\{t\}}\left[\sum_{\{b\}}{ }^{(0)} \prod_{\langle i j\rangle}{ }^{(0)} u^{b_{i j}}\right]\left[\sum_{\{b\}}{ }^{(1)} \prod_{\langle i j\rangle}{ }^{(1)} u^{b_{i j}} \prod_{k=1}^{n_{c}^{(1)}}(q-1)\right] \\
& \times\left[\sum_{\{b\}}{ }^{(2)} \prod_{\langle i j\rangle}{ }^{(2)}\left(1-b_{i j}\right)\right],
\end{aligned}
$$

where the clusters of color 1 are labeled $1,2, \ldots, n_{c}^{(1)}$. Execution of the type 0 and 2 sums and rewriting the type 1 sum yields the partition sum expressed in site variables, and bond variables only of type 1 :

$$
Z_{t b}=Z_{t b 1} \equiv \sum_{\{t\}}\left[\prod_{\langle i j\rangle}{ }^{(0)}(1+u)\right] \sum_{\{b\}}{ }^{(1)}\left[\prod_{k=1}^{n_{c}^{(1)}}(q-1) u^{n_{b}^{(k)}}\right] .
$$

Just as Eq. (2) describes the probability distribution of bond configurations of the random-cluster model, Eq. (10) represents the probability distribution of a system with both site variables $t_{i}=0,1$ and bond variables $b_{i j}$ between nearestneighbor sites of type 1 . The random selection of clusters of color 0 with probability $1 / q$ leads to the ensemble of Eq. (8) which describes a system of both site and bond variables. A partial summation on bond variables then leads to Eq. (10). In a Monte Carlo application of this mapping one makes use of the fact that the terms in the partition sums are proportional to the probability corresponding configuration. Subsequent assignments of values to random variables in the Monte Carlo procedure are decided such that the resulting probability of each configuration is in agreement with the partition function as expressed in the pertinent variables. This guarantees that the equilibrium distribution is always maintained. Thus starting from a bond configuration drawn from the equilibrium ensemble of Eq. (2):

(1) assign color 0 to each cluster with probability $1 / q$;

(2) erase all bonds $b_{i j}=1$ between type- 0 sites;

(3) choose new bonds $b_{i j}=1$ between type- 0 sites with probability $u /(u+1)$;

(4) form clusters on the type-0 sites;

(5) erase the color variables.

Here we have stochastically executed the step from Eqs. (2)-(7), followed by the steps leading to Eq. (10); and then, in reverse order, back to Eq. (2). This leads to a new bond configuration that again satisfies the equilibrium statistics of Eq. (2). We note that these steps resemble the SwendsenWang procedure; a difference is that one here uses 2 instead of $q$ colors, and that they are not treated equivalently. The use of a probability $1 / q$ restricts the useful range of the algorithm to $q>1$. The above description of the algorithm is given such as to closely follow the mapping; the actual procedure is even simpler because it is not necessary to keep track of the bond variables $b_{i j}$. These variables are only needed during the cluster formation process. The information to which cluster a site belongs is stored as an integer that is unique for each cluster.

\section{TESTS AND APPLICATION}

We have tested the three algorithms under investigation by comparing their numerical results mutually and, for $q=2$, with those of conventional algorithms. The results agree within the statistical errors.

\section{A. Application to specific-heat calculation}

To illustrate the use of the Monte Carlo algorithms under consideration, we have calculated the Potts specific heat for the random-cluster model on the square lattice for two noninteger values $q$. These are $q=4 \cos ^{2}(7 \pi / 22)=1.169 \cdots$ and $q=4 \cos ^{2}(5 \pi / 14)=0.753 \cdots$. The dimensionless specific heat (specific heat divided by $N k_{B}$ ) is here defined on the basis of differentiation of the free energy density $\ln Z$ to the Potts coupling $K$, with $Z$, e.g., defined as in Eq. (2):

$$
C \equiv \frac{K^{2}}{N} \frac{\partial^{2} \ln Z}{\partial K^{2}}
$$

with $\partial / \partial K=e^{K} \partial / \partial u$. Since the the sum $n_{b}$ of the bond variables is conjugate to $\ln u$, the specific heat can be obtained from the fluctuations of $n_{b}$. It is sufficient to sample the first two moments of $n_{b}$ :

$$
C=\frac{K^{2}}{N}\left[\frac{(u+1)^{2}}{u^{2}}\left(\left\langle n_{b}^{2}\right\rangle-\left\langle n_{b}\right\rangle-\left\langle n_{b}\right\rangle^{2}\right)+\frac{u+1}{u}\left\langle n_{b}\right\rangle\right] .
$$

The value of the temperature exponent of the Potts model is known as a function of $q$. This expression was conjectured by den Nijs [21]; see also Ref. [7]. For the specific-heat exponent $\alpha$ this expression leads to $\alpha=4 / 3-2$ / $[3-6 \arccos (\sqrt{q} / 2) / \pi]$. This formula allows us to select the value of $q$ corresponding to a given value of $\alpha$.

We first simulated the $q=4 \cos ^{2}(7 \pi / 22)$ Potts model, which has a specific-heat exponent $\alpha=-1 / 2$. We have calculated the Potts specific heat $C$ of the square-lattice model in a suitable temperature range, and obtained the curve shown in Fig. 1, which does indeed display a square-root type cusp as implied by $\alpha=-1 / 2$. During these simulations we found that the cluster algorithm was the most efficient one, i.e., produced a more accurate result in a given computer time. The results in Fig. 1 are those generated by the cluster algorithm.

Next, we simulated the square-lattice $q=4 \cos ^{2}(5 \pi / 14)$ Potts model, which has a specific-heat exponent $\alpha=-1$. We have calculated the Potts specific heat and obtained the curve shown in Fig. 2, which does indeed display a pronounced kink as implied by $\alpha=-1$. The results in Fig. 2 are those generated by the local bond update algorithm, because it became clear that it was more efficient than the reweighting algorithm.

These two figures, together with the well-known logarithmic divergence of the specific heat for $q=2$, illustrate that the 


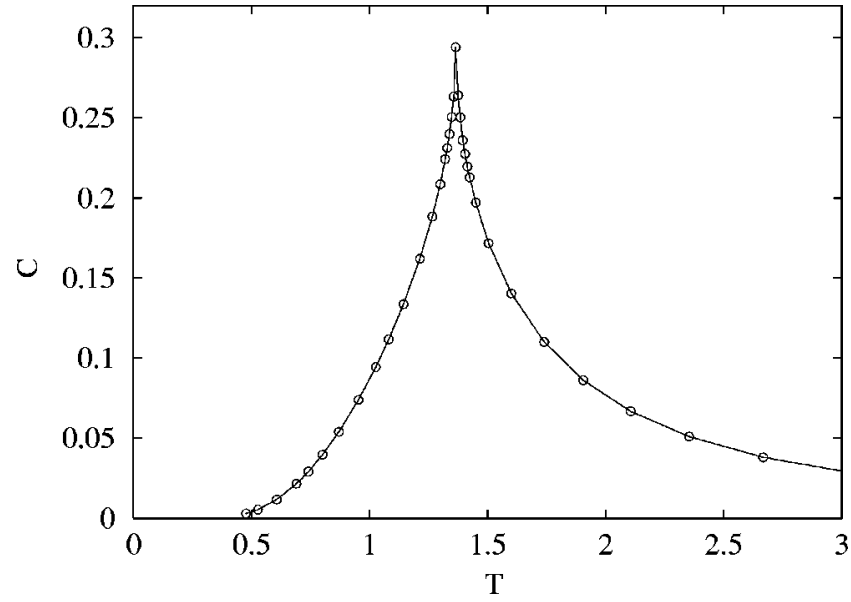

FIG. 1. Dimensionless Potts specific heat $C$ of the $q$ $=4 \cos ^{2}(7 \pi / 22)=1.169 \cdots$ random-cluster model versus temperature $T=1 / K$. The statistical errors do not exceed the size of the data points. The data points are extrapolations of finite-size data in the range $6 \leqslant L \leqslant 384$, obtained by means of the cluster Monte Carlo algorithm. The finite-size data converge exponentially except at the critical point where power-law behavior occurs. Satisfactory convergence was found for all $T$ except in very narrow ranges $(\Delta T$ $\approx 0.02$ ) on both sides of the critical point.

critical singularity becomes less strong when $q$ decreases. The use of $K$ as the temperature parameter facilitates a comparison with the results for the integer- $q$ Potts model. The negative specific heat for $q=4 \cos ^{2}(5 \pi / 14)<1$ reflects the fact that the Potts energy per bond decreases with temperature: the reduced energy is $K$ in the ordered state and $K / q$ in the disordered one. This illustrates the unphysical nature of the Potts model for $q<1$. We note, however, that the

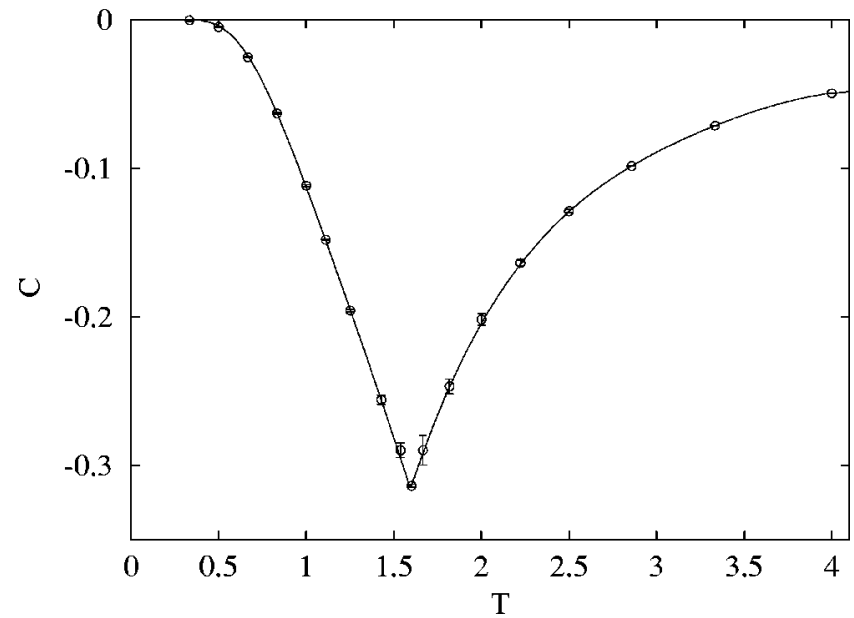

FIG. 2. Dimensionless Potts specific heat $C$ of the $q$ $=4 \cos ^{2}(5 \pi / 14)=0.753 \cdots$ random-cluster model versus temperature $T=1 / K$. The statistical errors are larger than in the preceding figure, in some cases they exceed the symbol size. The data points are extrapolations of finite-size data in the range $4 \leqslant L \leqslant 40$, obtained by means of the local bond-update Monte Carlo method. The finite-size data converge exponentially except at the critical point where power-law behavior occurs. Satisfactory convergence was found for all $T$ except in narrow ranges $(\Delta T \approx 0.2)$ on both sides of the critical point. random-cluster model is physical. For instance, its energy (not reduced) $E_{R C}=-k_{B} T\left\langle n_{c} \ln q+n_{b} \ln u\right\rangle$ is a well-behaved, increasing function of the temperature $T$ when the nonreduced parameters $k_{B} T \ln q$ and $k_{B} T \ln u$ are kept constant.

\section{B. Efficiency of the algorithms}

While the dynamical exponent is an important factor in the efficiency of an algorithm, it is not the only one. The degree of overlap between generated and target distributions is crucial in reweighting methods, and furthermore, the computer time per spin update may depend strongly on the system size. From a practical point of view one may be interested in the computer time needed to reach a result with a given statistical accuracy. Thus, to compare the performance of the three algorithms in a quantitative way, we have simulated the two-dimensional random-cluster model on the square lattice, and determined a dimensionless ratio similar to the Binder cumulant [22]. To this purpose we sampled powers of the cluster sizes

$$
S^{(m)} \equiv \sum_{k=1}^{n_{c}} s_{k}^{m},
$$

where $s_{k}$ is the size of the $k$ th cluster, for $m=2$ and 4 . Then the dimensionless ratio $Q$ is defined as

$$
Q \equiv \frac{\left\langle S^{(2)}\right\rangle^{2}}{\left\langle 3\left(S^{(2)}\right)^{2}-2 S^{(4)}\right\rangle},
$$

which, for the case $q=2$, reduces to the ratio of magnetization moments $\left\langle m^{2}\right\rangle^{2} /\left\langle m^{4}\right\rangle$. The computer time $t$ per lattice site needed to reach a statistical accuracy of $10^{-4}$ in $Q$ serves as an inverse measure of the efficiency. The results in terms of $t=\left(10^{4} \delta Q / L\right)^{2} t_{R}$, where $t_{R}$ is the CPU time of a run in seconds, $\delta Q$ is the statistical error in $Q$, and $L$ is the linear system size, are shown in Fig. 3. These results indicate that the cluster algorithm is more efficient than the other two, increasingly so for larger system sizes. The reweighting method appears to become rapidly inefficient with increasing system sizes. Here one may remark that a simple statistical analysis of the probability that the Monte Carlo algorithm generates a state in the center of the target distribution yields factors in which $N$ appears in the exponent. This argument thus indicates that the data for $t$ obtained by the reweighting method increase exponentially with $L^{2}$.

The interpretation of the results in Fig. 3 still requires some reservation. First, the reweighting method naturally becomes more efficient when $q$ approaches 1 . Nevertheless, the data shown are clear enough to indicate that the useful range of $q$ is quite narrow for the reweighting method. Second, our version of the local bond-update algorithm is relatively simple and forms clusters, instead of tracing their perimeter as in Sweeny's version. Since the fractal dimension of the perimeter is smaller than that of the cluster itself, Sweeny's version is expected to be more efficient than ours for sufficiently large system sizes, but at the expense of a more complicated code. Given the simplicity and efficiency of the cluster algorithm, we consider it the best choice for the investigation of $q>1$ models. 


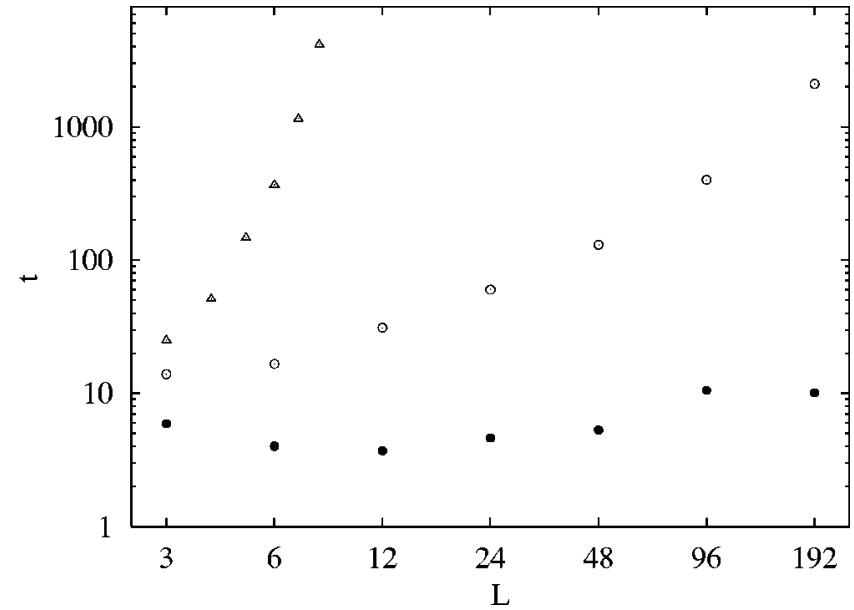

FIG. 3. Computer time usage $t$ of the three algorithms for the simulation of the continuous- $q$ random-cluster model, versus linear system size $L$. The time $t$ is determined as the computer time in seconds per lattice site required to reach an accuracy of $10^{-4}$ in the dimensionless ratio $Q$ of the random-cluster representation of the critical $q=2$ Potts model. The data points apply to the statistical reweighting method $(\triangle)$, to the local bond update method $(\bigcirc)$, and to cluster updates $(\bullet)$.

\section{Dynamic exponent of the cluster algorithm}

As mentioned in the Introduction, the reweighting method does not suffer, at least formally, from any critical slowing down, and thus its dynamic exponent is $z=0$. The dynamic exponent of the local bond-update method has recently been investigated by Wang et al. [17] for $q=2$ and 3. Their analysis, apparently more accurate than earlier investigations $[14,15]$, reported nonzero but still rather small values of $z$ that are, depending on the value of $q$, comparable with, or even somewhat smaller than those of the Swendsen-Wang algorithm. For $q \rightarrow 1$ one expects $z \rightarrow 0$ because the bond variables become independent. To evaluate the dynamic universality class of the cluster algorithm for continuous $q$, we have determined the dynamic exponent $z$ for three different values of $q$, on the basis of simulations of $L \times L$ square lattices with sizes $L=6,12, \ldots, 160$. We sampled the energy and determined its autocorrelation times $\tau_{L}$, in units of cluster steps as described in Sec. II, from least-squares fits to the exponentially decaying autocorrelation function. The results are shown in Fig. 4. We have analyzed their $L$ dependence as $\tau_{L} \simeq L^{z}$ by means of least-squares fits. We obtain $z=0.08(1)$ for $q=4 \cos ^{2}(7 \pi / 22)$, and $z=0.551$ (8) for $q=3$. The result for $q=3$ is consistent with an existing result for the Swendsen-Wang algorithm, namely $z=0.56(1)$ [17], but it is larger than the result of a more detailed study [23], using system sizes up to $L=1024$, which is $z=0.515(6)$. The question thus arises whether the difference with the continuous- $q$ cluster algorithm, which amounts to a few standard deviations, indicates that the dynamic universality classes of the two algorithms are different. We do not consider the numerical evidence to be sufficient to reach such a conclusion: it was noted in Ref. [23] that the largest system sizes ( $L$ $\geqslant 128$ ) led to a significantly smaller result in comparison with the smaller system sizes. This suggest the presence of

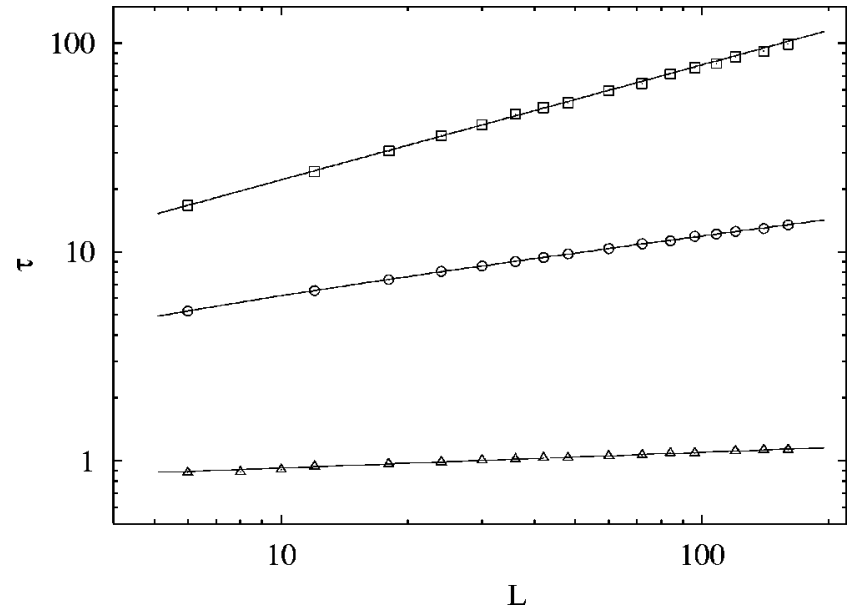

FIG. 4. Autocorrelation times $\tau$ versus system size $L$ for three critical Potts models: $q=1.169 \cdots(\triangle), q=2(\bigcirc)$, and $q=3(\square)$ on logarithmic scales. These results were obtained using the continuous- $q$ cluster algorithm. The statistical errors do not exceed the size of the data points.

slowly converging correction terms in the autocorrelation times; such corrections could also be present in our results for the continuous- $q$ cluster algorithm, and thus also explain the difference with our value of $z$.

Especially for $q=2$ the autocorrelation times are not well described by a single power law; the fits suggest the presence of a second term proportional to $L^{z^{\prime}}$ with $z^{\prime} \approx-0.4$. Allowing for such a contribution we obtain $z=0.254$ (10) for $q=2$, which is consistent with a result $z=0.25(1)$ [24] for the Swendsen-Wang algorithm, but larger than a more recent determination $[9,25]$ which led to $z=0.222(7)$ on the basis of system sizes up to $L=512$. We found that our $q=2$ result for $z$ depends considerably on the choice of the fit formula and the range of $L$. Instead of adding a second term, one can choose to skip the smallest system sizes in order to obtain an acceptable value of the residual $\chi^{2}$. For system sizes in the range $60 \leqslant L \leqslant 160$ we thus find $z=0.265(5)$. This number, as well as $\chi^{2}$, increases when the lower limit of the $L$ range is decreased (these numbers agree better with earlier determinations $[8,26]$ which are close to $z=0.3)$. Under these circumstances, we do not assign much significance to the differences between the reported values of $z$ for the SwendsenWang algorithm and the continuous- $q$ cluster algorithm. These differences are of the same order as those between different results reported for the Swensen-Wang algorithm and may be attributed to unresolved corrections to scaling.

\section{ACKNOWLEDGMENTS}

We are indebted to J. R. Heringa and J.-S. Wang for valuable discussions. This research was supported by the Dutch FOM Foundation ("Stichting voor Fundamenteel Onderzoek der Materie") which is financially supported by the NWO ("Nederlandse Organisatie voor Wetenschappelijk Onderzoek"). We acknowledge the hospitality of the Institute for Mathematical Sciences of the National University of Singapore (2004), where we learned about the present status of continuous- $q$ Monte Carlo algorithms. 
[1] R. B. Potts, Proc. Cambridge Philos. Soc. 48, 106 (1952).

[2] F. Y. Wu, Rev. Mod. Phys. 54, 235 (1982).

[3] P. W. Kasteleyn and C. M. Fortuin, J. Phys. Soc. Jpn. 26, 11 (1969).

[4] R. J. Baxter, S. B. Kelland, and F. Y. Wu, J. Phys. A 9, 397 (1976).

[5] R. J. Baxter, Phys. Rev. Lett. 26, 832 (1971).

[6] R. J. Baxter, J. Phys. C 6, L445 (1973).

[7] B. Nienhuis, in Phase Transitions and Critical Phenomena, edited by C. Domb and J. L. Lebowitz (Academic, London, 1987), Vol. 11, p. 55.

[8] R. H. Swendsen and J.-S. Wang, Phys. Rev. Lett. 58, 86 (1987).

[9] G. Ossola and A. D. Sokal, Nucl. Phys. B 691, 259 (2004).

[10] B. Nienhuis, A. N. Berker, E. K. Riedel, and M. Schick, Phys. Rev. Lett. 43, 737 (1979).

[11] B. Nienhuis, E. K. Riedel, and M. Schick, Phys. Rev. B 23, 6055 (1981).

[12] S. Grollau, M. L. Rosinberg, and G. Tarjus, Physica A 296, 460 (2001).
[13] Y. Deng, H. W. J. Blöte, and B. Nienhuis, Phys. Rev. E 69, 026114 (2004).

[14] M. Sweeny, Phys. Rev. B 27, 4445 (1983).

[15] F. Gliozzi, Phys. Rev. E 66, 016115 (2002).

[16] X. J. Li and A. D. Sokal, Phys. Rev. Lett. 63, 827 (1989).

[17] J.-S. Wang, O. Kozan, and R. H. Swendsen, Phys. Rev. E 66, 057101 (2002)

[18] C.-K. Hu, Phys. Rev. Lett. 69, 2739 (1992).

[19] J. R. Heringa and H. W. J. Blöte, Phys. Rev. Lett. 70, 2044 (1993).

[20] L. Chayes and J. Machta, Physica A 254, 477 (1998).

[21] M. P. M. den Nijs, J. Phys. A 12, 1857 (1979).

[22] K. Binder, Z. Phys. B: Condens. Matter 43, 119 (1981).

[23] J. Salas and A. D. Sokal, J. Stat. Phys. 87, 1 (1997).

[24] P. D. Coddington and C. F. Baillie, Phys. Rev. Lett. 68, 962 (1992).

[25] J. Salas and A. D. Sokal, e-print cond-mat/9904038v1, Sec. 6.

[26] C. F. Baillie and P. D. Coddington, Phys. Rev. B 43, 10617 (1991). 